\title{
El efecto modulador del clima organizacional en la influencia de la comunicación de funciones directivas sobre el conflicto de rol"
}

\author{
Modulatory Effect of Organizational Climate in the Influence \\ of Communication Leadership Roles on Role Conflict
}

Recibido: 10 de enero de 2014 | Aceptado: 02 de febrero de 2016

\author{
Pedro Antonio Díaz-Fúnez ** \\ Miguel ÁNGel MAÑAS-RODRÍGUEZ \\ Vicente PeCino-Medina \\ CARMEn María Salvador-FERrer \\ JUAN MANUEL LLOPIS-MARÍN \\ Universidad de Almería, España
}

doi : 10.11144/Javeriana.upsy15-1.emco

Para citar este artículo: Díaz-Fúnez, P. A., MañasRodríguez, M. Á., Pecino-Medina, V., SalvadorFerrer, C. M., \& Llopis-Marín, J. M. (2016). El efecto modulador del clima organizacional en la influencia de la comunicación de funciones directivas sobre el conflicto de rol. Universitas Psychologica, 15(1), 383-394. http://dx.doi.org/10.11144/ Javeriana.upsy15-1.emco

* Artículo de investigación, financiado por las Cooperativas Hortofrutícolas de Almería.

** Grupo de investigación de psicología del trabajo, las organizaciones y los recursos humanos. Carretera de Sacramento s/n. 04120 Almería (España). Tel. 950015405 y638140104.Email: pfunez@ual.es (IPTORA HUM-923).

\section{RES U MEN}

Este trabajo analiza el papel que ejerce el clima organizacional como elemento modulador entre la comunicación de funciones directivas y el conflicto de rol. En el presente estudio han participado 315 trabajadores de cooperativas hortofrutícolas almerienses. Los resultados generales mostraron que el clima es un elemento modulador de la relación entre la comunicación de funciones directivas y el conflicto rol. En concreto, indicaron que la influencia de la comunicación de funciones directivas hace disminuir el conflicto de rol y este efecto se incrementa cuando aumenta la percepción de clima de apoyo, clima de innovación y clima de orientación a reglas. Se discuten las implicaciones prácticas del estudio, junto con las limitaciones y sugerencias para futuras investigaciones derivadas del mismo.

Palabras clave

comunicación de funciones directivas; conflicto de rol; clima organizacional; moderación; sector hortofrutícola

\section{A B S T R A C T}

This paper analyzes the role exerted by the organizational climate as a modulator of communication between leadership and role conflict. To do this, the study involved 315 workers of cooperatives in horticulture. The overall results showed that the weather is an element modulator of communication regarding leadership and role conflict. Specifically, the data indicated that the negative influence of communication in leadership on role conflict increases with increasing perceived supportive climate, weather and climate innovation orientation rules, although with lower modulation effects the first two. We discuss the practical implications of the study, along with the limitations and suggestions for future research arising therefrom. Keywords

Communication leadership; Role Conflict; Organizational Climate; Moderation Horticulture firm 


\section{Introducción}

En la actualidad, el entorno laboral es muy distinto del existente hace unos años a causa de la crisis financiera global. Esto ha provocado el aumento de las consecuencias negativas asociadas al trabajo, entre las que destaca el aumento de los riesgos psicosociales y enfermedades asociadas con la vida laboral de los trabajadores, tales como el estrés, el síndrome de estar quemado (burnout) y la depresión, entre otros (MacKenzie, Garavan, \& Carbery, 2012; Tomasina, 2012).

Desde los primeros estudios, la investigación psicosocial del entorno organizacional ha insistido en destacar el recurso humano como elemento clave para alcanzar la eficiencia organizacional (Likert, 1961; McGregor, 1960). Estos resultados han conducido a pensar en una mayor atención a la gestión de este recurso y en la implementación de prácticas innovadoras a la hora de gestionarlo, siendo esta una de las principales líneas que se está investigando actualmente a la hora de reducir los riesgos psicosociales en las organizaciones (Salanova, Llorens, Cifre, \& Martínez, 2012).

Partiendo del nuevo modelo de análisis del efecto de las prácticas de recursos humanos sobre las per- sonas, desarrollado por Albrecht, Bakker, Gruman, Macey y Saks (2015), este trabajo trata de mostrar el efecto modulador y central del clima organizacional a la hora de reducir los riesgos psicosociales. En concreto, se trata de mostrar el efecto de modulación que presenta el clima organizacional en la influencia de las habilidades directivas sobre el conflicto de rol.

\section{Marco teórico}

Podemos definir el clima organizacional como el conjunto de percepciones compartidas, relacionadas con las políticas, prácticas y procesos que una organización recompensa, apoya y espera (Schneider, \& Reichers, 1983). El modelo teórico para medir clima organizacional, utilizado en este trabajo es el desarrollado por De Witte y De Cock (1986) a partir de la "aproximación de valores en competencia” propuesta por Quinn y Rohrbaugh (1983). En este modelo, cruzando los valores la orientación de la organización (interna-externa) y los de la estructura (flexibilidad-control), surgen cuatro valores en conflicto: el clima de apoyo en contraposición al clima de orientación a reglas y el clima de innovación en oposición al clima de orientación a metas.

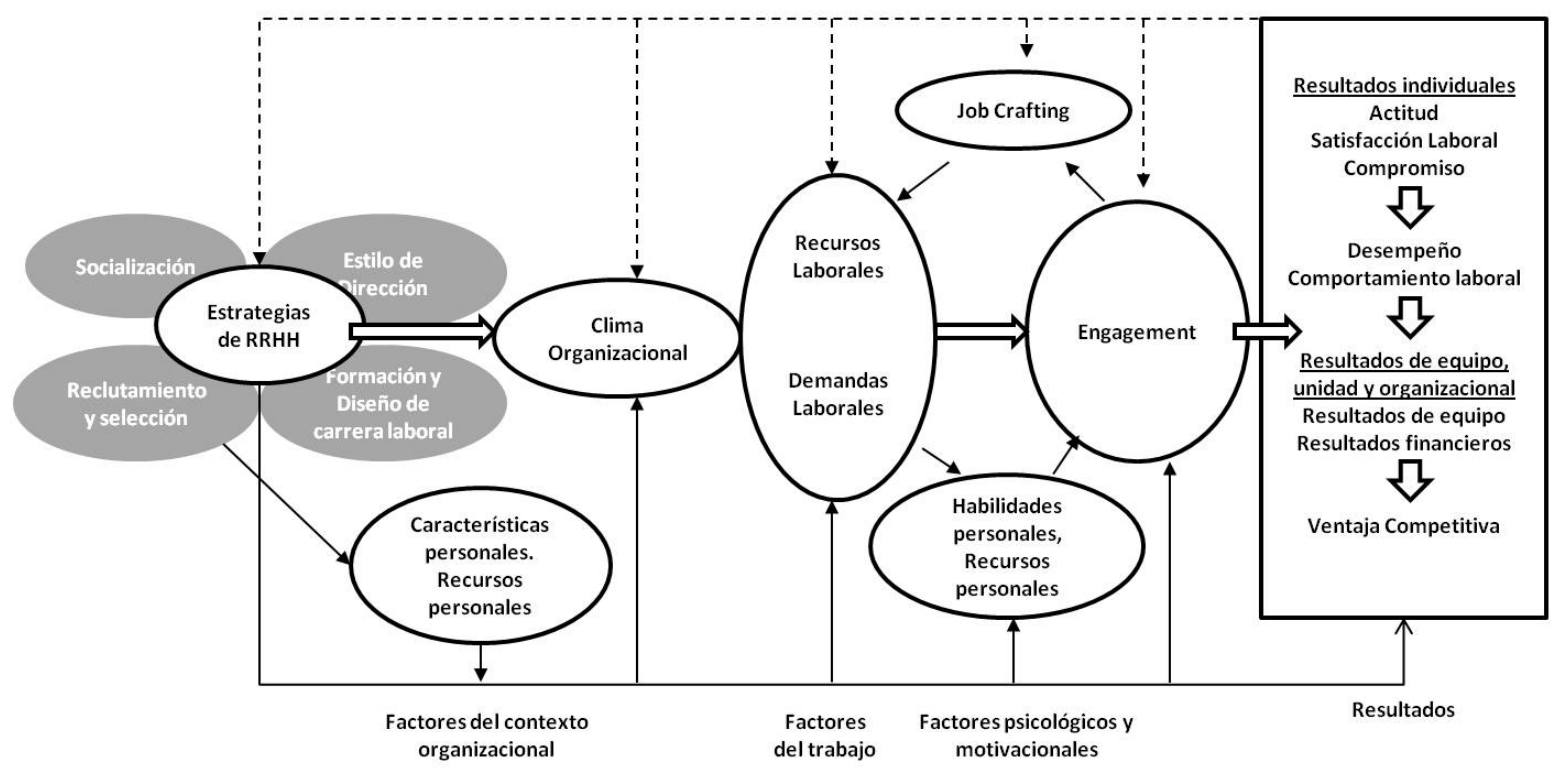

Figura 1. Modelo integrado de RRHH basado en Engagement

Fuente: adaptado de Albrecht et al., 2015 
A la hora de plantear las hipótesis de trabajo se ha partido del modelo teórico integrado de recursos humanos y engagement de Albrecht et al. (2015) y una actualización del modelo de estudio de clima organizacional de Kopelman, Brief y Guzzo (1990). Así, la comunicación de funciones directivas es definida como un factor del contexto organizacional que influye directamente tanto sobre el clima organizacional como sobre los factores del trabajo, como es el caso del conflicto de rol.

Entre los elementos que pueden afectar el entorno social de la organización, la comunicación de funciones directivas se define dentro de las conductas del superior inmediato, cuyo fin es clarificar las responsabilidades y objetivos de desempeño (Yukl, 2006). Recientes trabajos de investigación han mostrando una relación directa, significativa y positiva entre estas variables (McMurray, Islam, Sarros, \& Pirola-Merlo, 2012; Pérez, Guzmán, \& Santa Cruz, 2014).

El conflicto de rol, por su parte, es una de las dimensiones que se incluye en el estrés de rol. Podemos definirlo como el grado de incongruencia o incompatibilidad de las expectativas del trabajador asociadas al desempeño del rol (House $\&$ Rizzo, 1972). Diversos estudios afirman que comportamientos de apoyo de los directivos a los empleados están asociados con bajos niveles de conflicto de rol, demostrándose una influencia significativa y de signo negativo entre ambas variables (Babin \& Boles, 1996; House \& Rizzo, 1972; Teas, 1983). Entre esas conductas, autores como Johlke y Duhan (2001) señalaron que las prácticas de comunicación del supervisor están asociadas con el descenso de estrés de rol de los trabajadores, confirmando que la comunicación dirigida a visualizar las metas y los objetivos de las actividades del equipo de trabajo, a proporcionar información sobre los clientes y a informar sobre las posibilidades de promoción reducen la incertidumbre de los trabajadores y, por ende, el conflicto de rol.

Siguiendo el modelo de Albrecht et al. (2015), y apoyado en la revisión bibliográfica previa, este trabajo pretende demostrar que el clima organizacional interviene como variable moduladora en la relación e influencia entre la comunicación de funciones directivas y el conflicto de rol, esto es, el clima organizacional alterará la dirección o intensidad de la relación e influencia entre las variables citadas (Calvete, 2008; Cohen, Cohen, West, \& Aiken, 2003; Frazier, Tix, \& Barron, 2004). Por todo ello, se proponen las siguientes hipótesis:

Hipótesis 1. La dimensión de clima de apoyo moderará de forma significativa y relevante la relación e influencia de la comunicación de funciones directivas y el conflicto de rol.

Hipótesis 2. La dimensión de clima de innovación moderará de forma significativa y relevante la relación e influencia de la comunicación de funciones directivas y el conflicto de rol.

Hipótesis 3. La dimensión de clima de orientación a metas moderará de forma significativa y relevante la relación e influencia de la comunicación de funciones directivas y el conflicto de rol.

Hipótesis 4. La dimensión de clima de orientación a reglas moderará de forma significativa y relevante la relación e influencia de la comunicación de funciones directivas y el conflicto de rol.

\section{Método}

\section{Participantes}

La muestra está compuesta por un total de 315 personas dedicadas al sector hortofrutícola, procedentes de tres organizaciones, lo que supone una tasa de respuesta del $89.3 \%$ del total de los empleados de estas organizaciones. En lo que respecta a la edad de la muestra, el mayor porcentaje (40\%) corresponde al intervalo de 26 y 35 años, seguido de un $33 \%$ de personas cuyas edades se encuentran comprendidas entre los 36 y 45 años, siendo la edad media de la muestra 33.4 años. En lo concerniente al sexo, encontramos que un $55 \%$ son mujeres y un $45 \%$ son hombres. Por último, en lo relacionado con los estudios recibidos, encontramos que un $32 \%$ tienen educación primaria, seguido de un $16 \%$ que tienen certificado de escolaridad, un $12 \%$ educación secundaria, otro $12 \%$ diplomatura y un $15 \%$ licenciatura, mientras que sólo un $3 \%$ señala no tener estudios. 


\section{Instrumentos}

A continuación, se exponen los distintos instrumentos de medida utilizados en el presente trabajo. La comunicación de funciones directivas fue medida a través de una escala desarrollada por Peiró y González-Romá (1994) para el estudio sobre los correlatos psicológicos del absentismo en el personal del Servicio Valenciano de Salud, basándose en la taxonomía de Yukl y Van Fleet (1992) sobre conductas directivas. La escala está formada por tres ítems, donde se les pedía a los sujetos que evaluaran el grado de comunicación que tenía su jefe o superior inmediato en relación a la transmisión de información referente a políticas y prácticas de la organización, trabajo a desempeñar y otros temas de interés. Se utilizó una escala de respuesta tipo Likert, con valores que oscilaban entre 1 (muy en desacuerdo) y 5 (muy de acuerdo). Un ejemplo de ítem de esta escala es nuestro superior nos informa de las políticas y prácticas de la organización. La puntuación obtenida en el coeficiente $\alpha$ de Cronbach es de 0.913.

El clima organizacional ha sido medido mediante el cuestionario FOCUS-93 (Van Muijen et ál., 1994). Este consta de 40 ítems utilizando una escala de respuesta que oscila entre 1 (nadie/nunca) y 6 (todos/siempre). El instrumento valora cuatro dimensiones: clima de apoyo (ocho ítems y $\alpha$ de Cronbach de 0.869), clima de innovación (doce ítems y $\alpha$ de Cronbach de 0.784), clima de orientación a reglas (seis ítems y $\alpha$ de Cronbach de 0.728) y clima de orientación a metas (catorce ítems y $\alpha$ de Cronbach de 0.779). A continuación se presentan como ejemplo algunos de los ítems: Ítem de Apoyo: "ilos directores manifiestan su preocupación por los problemas personales de sus empleados?". Item de O. Metas: "icon qué frecuencia se mide su rendimiento?". Ítem de O. Reglas: "ila comunicación en la empresa sigue la estructura jerárquica?". Ítem de Innovación "iel ambiente externo demanda cambios en su trabajo?".

El conflicto de rol se ha medido a través del cuestionario de Rizzo, House y Lirtzman (1970) adaptado por Peiró, Meliá, Torres y Zurriaga (1986). El formato de respuesta es tipo Likert de cinco alternativas de respuesta que oscilan entre 1 (muy en desacuerdo) y 5 (muy de acuerdo). Está compuesto por oocho ítems (p. ej., recibo demandas incompatibles de dos o más personas) y tiene un coeficiente $\alpha$ de Cronbach de 0.841 .

\section{Análisis}

Como análisis previo, se realizaron análisis descriptivos y de correlación. Posteriormente, las hipótesis de este trabajo se pusieron a prueba mediante análisis de regresión (Cohen et al., 2003). Todos los análisis se realizaron mediante el paquete estadístico SPSS (versión 18.0).

\section{Resultados}

\section{Análisis descriptivos}

A continuación, se indican los resultados descriptivos y las correlaciones entre las variables del estudio

TABLA 1.

Descriptivos y correlaciones

\begin{tabular}{|c|c|c|c|c|c|c|c|c|}
\hline Variables & M & $\mathrm{Sd}$ & 1 & 2 & 3 & 4 & 5 & 6 \\
\hline 1. Comunicación F.D. & 3.0452 & 1.31648 & - & & & & & \\
\hline 2. Clima de apoyo & 2.9923 & 1.11070 & $0.701 * * *$ & - & & & & \\
\hline 3. Clima de innovación & 3.0859 & 0.76150 & $0.530 * * *$ & $0.737 * * *$ & - & & & \\
\hline 4. Clima de metas & 3.0463 & 0.77852 & $0.433 * * *$ & $0.584 * * *$ & $0.687 * * *$ & - & & \\
\hline 5. Clima de reglas & 3.2019 & 0.92723 & $0.616 * * *$ & $0.742 * * *$ & $0.654 * * *$ & $0.600 * * *$ & - & \\
\hline 6. Conflicto de rol & 3.0339 & 0.95544 & $-0.279 * * *$ & $-0.200 * * *$ & -0.104 & $-0.131^{*}$ & $-0.221 * * *$ & - \\
\hline \multicolumn{9}{|c|}{$\begin{array}{l}* * * \mathrm{p}<0.001 ; * * \mathrm{p}<0.01 ; * \mathrm{p}<0.05 \\
\text { Fuente: elaboración propia }\end{array}$} \\
\hline 386 & UNI & RSITAS P & YCHOLOGIC & A $\mid$ V. 15 & 5 | No. 1 & I ENER & D-MARZO & 2016 \\
\hline
\end{tabular}


(Tabla 1). Respecto a las correlaciones se destacan las relaciones significativas y positivas de "comunicación de funciones directivas" con todas las dimensiones de clima organizacional y la relación negativa con "conflicto de rol". Asimismo, las correlaciones entre las dimensiones de clima organizacional y "conflicto de rol" son significativas y negativas, excepto con "clima de innovación”, que no muestra relación significativa.

\section{Análisis de regresión}

Para poner a prueba el objetivo del estudio, y analizar de qué modo el clima organizacional modera la relación entre la comunicación de funciones directivas y el conflicto de rol, se han llevado a cabo cuatro ecuaciones de regresión jerárquica (Tabla 2).

Como se puede comprobar en el primer paso de la Tabla 2, el modelo que incluye la variable comunicación de funciones directivas como variable antecedente del conflicto de rol es significativo ( $F=$ $25.982 ; p<0.001)$ y explica un $7.8 \%$ de la varianza del conflicto de rol. En el segundo paso, la introducción de las variables comunicación de funciones directivas y clima de apoyo también es significativo $(F=13.011 ; p<0.001)$, pero el clima de apoyo no muestra relación significativa con conflicto de rol,

TABLA 2.

Efectos de moderación del Clima de apoyo sobre la relación entre comunicación de funciones directivas y conflicto de rol

\begin{tabular}{ccccc}
\hline & & $\beta$ & $\mathrm{F}$ & $\mathrm{R} 2$ \\
\hline Paso 1 & CFD & $-0.279 * * *$ & $25.982 * * *$ & $0.078^{* * *}$ \\
Paso 2 & CFD & $-0.261 * * *$ & $13.011 * * *$ & $0.078 * * *$ \\
& Clima de apoyo & -0.026 & & \\
Paso 3 & CFD & $-0.279 * * *$ & $12.350 * * *$ & $0.108 * * *$ \\
& Clima de apoyo & -0.025 & & \\
& CFD x Clima de apoyo & $-0.178^{* *}$ & & \\
\hline
\end{tabular}

$* * * \mathrm{p}<0.001 ; * * \mathrm{p}<0.01 ; * \mathrm{p}<0.05$. CFD: comunicación de funciones directivas Fuente: elaboración propia

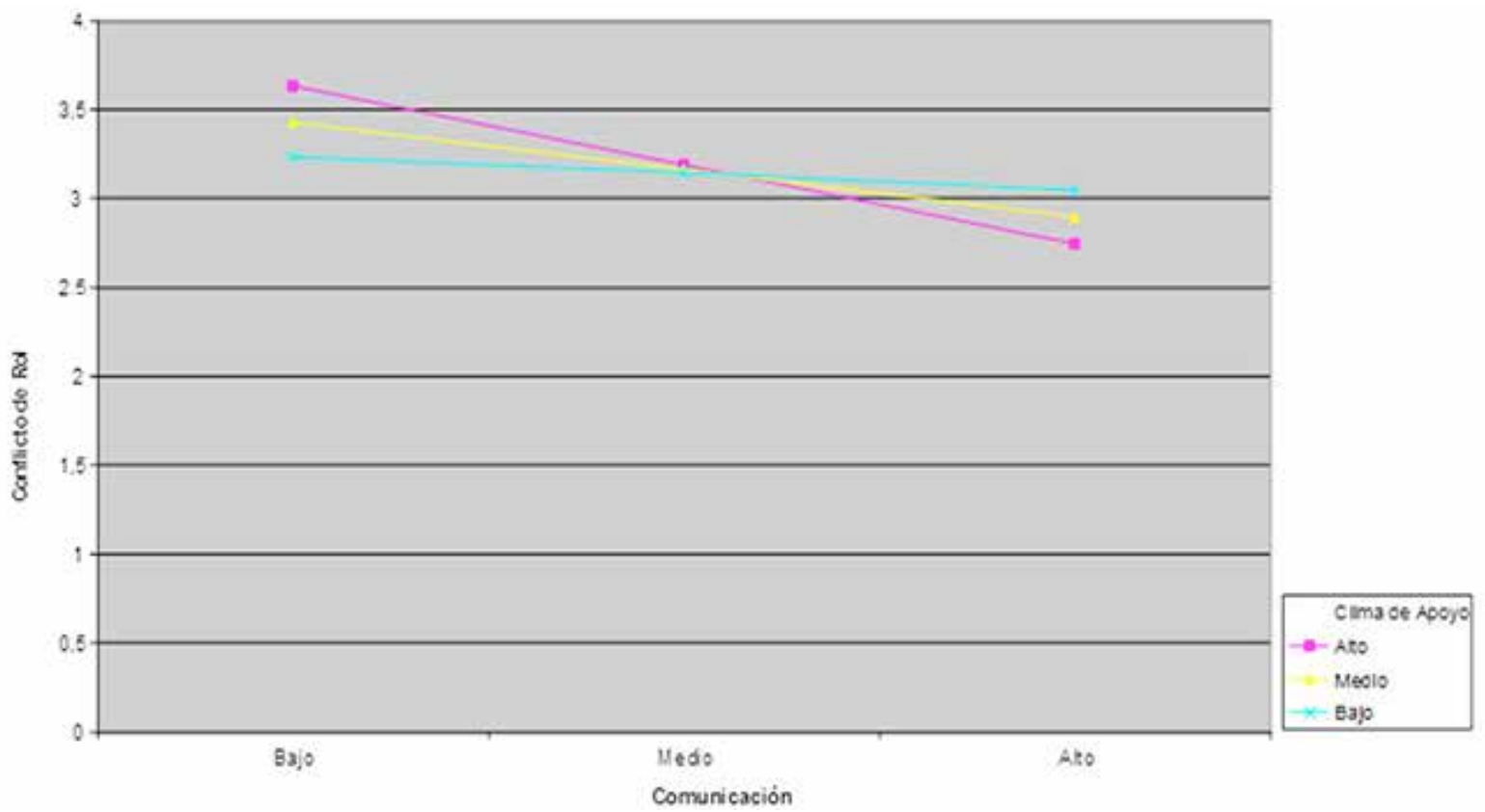

Figura 3. Efectos de modulación del clima de apoyo sobre la relación entre comunicación de funciones directivas y conflicto de rol Fuente: elaboración propia 
por lo que no se produce mejora en la capacidad predictiva del modelo. Finalmente, la introducción del término de interacción (comunicación de funciones directivas $\mathrm{x}$ clima de apoyo) también es significativa $(F=12.350 ; p<0.001)$, además el porcentaje de varianza explicada del conflicto de rol aumenta de forma significativa hasta el 10,8\% y se distinguen dos interacciones significativas que relacionan la comunicación de funciones directivas $(\beta:-0.279 ; p<0.001)$ y la interacción entre comunicación de funciones directivas y clima de apoyo $(\beta:-0.178 ; p<0.001)$ con el conflicto de rol, siendo este último efecto menor que el mostrado por la variable independiente de forma aislada.

Si se analiza esta interacción (Figura 3), se puede observar que en situaciones de bajo clima de apoyo, el aumento de las conductas de comunicación de funciones directivas tiene un efecto leve sobre el conflicto de rol en los trabajadores, mientras que con niveles altos de clima de apoyo, el aumento de la comunicación de funciones directivas tiene un efecto relevante de reducción del conflicto de rol.

En la Tabla 3 se presenta la ecuación de regresión que incluye el clima de innovación como variable moduladora de la relación entre la comunicación de funciones directivas y el conflicto de rol.

Como se puede comprobar en el primer paso de la Tabla 3, el modelo es significativo $(F=25.982$; $p<0.001$ ) y explica un $7.8 \%$ de la varianza del conflicto de rol. En el segundo paso, la introducción de las variables comunicación de funciones directivas y clima de innovación también es significativa $(F=13.245 ; p<0.001)$, pero aunque el clima de innovación no muestra relación significativa con el conflicto de rol, se produce una leve mejora en la capacidad predictiva del modelo (8\%). Finalmente, la introducción del término de interacción (comunicación de funciones directivas x clima de innovación) también es significativo $(F=10.490$; $p<0.001$ ), advirtiendo que existen dos interacciones significativas que relacionan la comunicación de funciones directivas $(\beta:-0.297 ; p<0.001)$ y la interacción entre comunicación de funciones directivas y clima de apoyo $(\beta:-0.119 ; p<0.001)$ con el conflicto de rol y que, además, el porcentaje de varianza explicada del conflicto de rol aumenta de forma significativa hasta el 9.4\%.

En la Figura 4 podemos observar cómo en contextos organizacionales con una alta orientación hacia la innovación, la existencia de elevados niveles de comunicación presenta los niveles de conflicto de rol más bajos. Por su parte, cuando la comunicación de funciones directivas es baja, ese mismo contexto orientado a potenciar la innovación en la organización es el que mayores niveles de conflicto de rol presenta.

TABLA 3.

Efectos de moderación del clima de innovación sobre la relación entre comunicación de funciones directivas y conflicto de rol $\beta$ $\mathrm{F}$

\begin{tabular}{|c|c|c|c|c|}
\hline & & $\beta$ & $\mathrm{F}$ & \\
\hline Paso 1 & CFD & $-0.279 * * *$ & $25.982 * * *$ & $0.078^{* * * *}$ \\
\hline Paso 2 & $\begin{array}{c}\text { CFD } \\
\text { Clima de innovación }\end{array}$ & $\begin{array}{c}-0.305 * * * \\
0.048\end{array}$ & $13.245 * * *$ & $0.080 * * *$ \\
\hline Paso 3 & $\begin{array}{c}\text { CFD } \\
\text { Clima de innovación } \\
\text { CFD x clima innovación }\end{array}$ & $\begin{array}{c}-0.297 * * * \\
0.061 \\
-0.119 *\end{array}$ & $10.490 * * *$ & $0.094 * * *$ \\
\hline
\end{tabular}

$* * * \mathrm{p}<0.001 ; * * \mathrm{p}<0.01 ; * \mathrm{p}<0.05$. CFD: comunicación de funciones directivas

Fuente: elaboración propia 


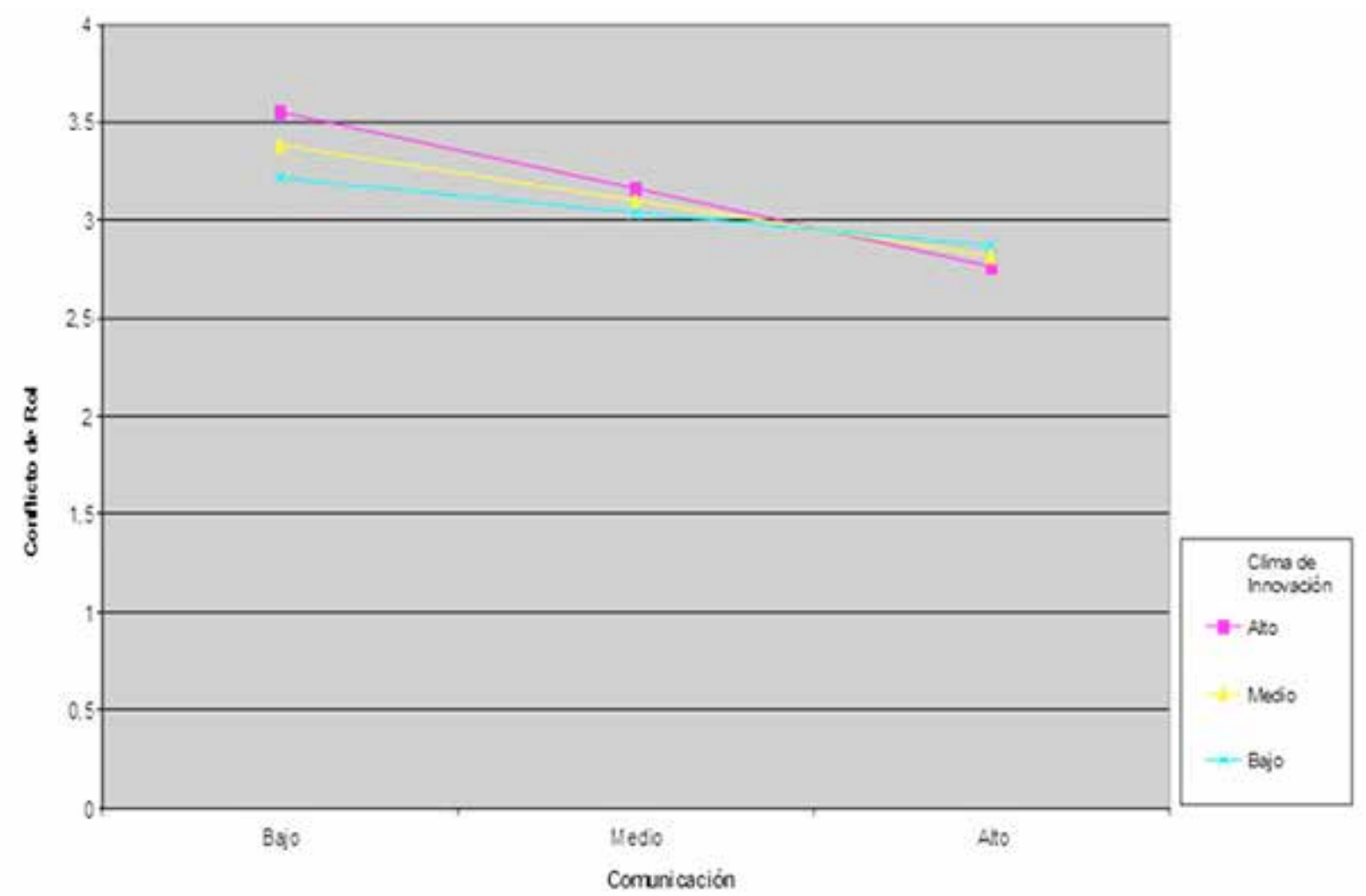

Figura 4. Efectos de modulación del clima de innovación sobre la relación entre comunicación de funciones directivas y conflicto de rol

Fuente: elaboración propia

La Tabla 4 muestra los resultados de la ecuación de regresión que incluye el clima de orientación a metas moderando los efectos de la comunicación de funciones directivas sobre el conflicto de rol.

Los resultados de la Tabla 4 muestran que, aunque en el paso 3 incluye el efecto de moderación, tiene efecto significativo $(F=9.665 ; p<0.001)$, no aumenta la capacidad explicativa respecto a los otros dos modelos anteriores (7.8\%), por lo que el clima de orientación a metas no presenta efecto de moderación significativo entre las variables comunicación de funciones directivas y conflicto de rol $(\beta=-0.094, n s)$.

La Tabla 5 presenta los resultados de la ecuación de regresión que contempla el clima de orientación a reglas como moduladora de la relación entre la comunicación de funciones directivas y el conflicto de rol.

En la Tabla 5 se observa, en el segundo paso, que la introducción de las variables comunicación de funciones directivas y clima de orientación a reglas es significativo $(F=13.717 ; p<0.001)$, pero aunque el clima de orientación a reglas no muestra relación significativa con el conflicto de rol, se produce una leve mejora en la capacidad predictiva del modelo (8.2\%). Finalmente, la introducción del término de interacción (comunicación de funciones directivas $\mathrm{x}$ clima de innovación) también es significativa $(F=12.961 ; p<0.001)$, advirtiendo que existen dos interacciones significativas que relacionan la comunicación de funciones directivas $(\beta=-0.225$; $p<0.001)$ y la interacción entre comunicación de funciones directivas y clima de orientación a reglas $(\beta=-0.176 ; p<0.001)$ con el conflicto de rol y que, además, el porcentaje de varianza explicada del conflicto de rol aumenta de forma significativa hasta el $11.3 \%$.

La representación gráfica de estos resultados (Figura 5) muestra como frente a una comuni- 
TABLA 4.

Efectos de moderación del clima de orientación a metas sobre la relación entre comunicación de funciones directivas y conflicto de rol

\begin{tabular}{ccccc}
\hline & & $\beta$ & $\mathrm{F}$ & \\
\hline Paso 1 & CFD & $-0.279^{* * *}$ & $25.982^{* * *}$ & $0.078^{* * *}$ \\
\hline \multirow{2}{*}{ Paso 2 } & CFD & $-0.274^{* * * *}$ & $12.972^{* * *}$ & $0.078^{* * *}$ \\
& Clima de metas & -0.013 & & $0.078^{* * *}$ \\
\hline Paso 3 & CFD & $-0.273^{* * *}$ & $9.665^{* * * *}$ & \\
& Clima de metas & 0.001 & & \\
& CFD x Clima de metas & -0.094 & & \\
\hline
\end{tabular}

$* * * \mathrm{p}<0.001 ; * * \mathrm{p}<0.01 ; * \mathrm{p}<0.05$. CFD: comunicación de funciones directivas Fuente: elaboración propia

TABLA 5.

Efectos de moderación del clima de orientación a reglas sobre la relación entre comunicación de funciones directivas y conflicto de rol

\begin{tabular}{|c|c|c|c|c|}
\hline & & $\beta$ & $\mathrm{F}$ & \\
\hline Paso 1 & CFD & $-0.279 * * *$ & $25.982 * * *$ & $0.078 * * *$ \\
\hline \multirow[t]{2}{*}{ Paso 2} & CFD & $-0.228 * * *$ & $13.717 * * *$ & $0.082 * * *$ \\
\hline & Clima de reglas & -0.083 & & \\
\hline Paso 3 & $\begin{array}{c}\text { CFD } \\
\text { Clima de reglas } \\
\text { CFD x Clima de reglas }\end{array}$ & $\begin{array}{c}-0.225 * * * \\
-0.078 \\
-0.176 * * *\end{array}$ & $12.961 * * *$ & $0.113 * * *$ \\
\hline
\end{tabular}

$* * * \mathrm{p}<0.001 ; * * \mathrm{p}<0.01 ; * \mathrm{p}<0.05$. CFD: comunicación de funciones directivas

Fuente: elaboración propia

cación de funciones directivas baja, la existencia de un contexto con una alta orientación a reglas presenta los mayores índices de conflicto de rol. Sin embargo, cuando la comunicación de funciones directivas alcanza los valores más altos y existe un alto clima de orientación a reglas, se producen los niveles más bajos de conflicto de rol en los trabajadores.

\section{Discusión y conclusiones}

El objetivo de este trabajo ha sido examinar el efecto modulador del clima organizacional en la relación entre la comunicación de funciones directivas y el conflicto de rol.

En la primera hipótesis se planteaba que el clima de apoyo moderaría de forma significativa y relevante la relación e influencia de la comu- nicación de funciones directivas y el conflicto de rol, los resultados obtenidos nos permiten confirmar esta hipótesis, pues el clima de apoyo moderó dicha relación e influencia, añadiendo un $3 \%$ de explicación de la varianza de conflicto de rol (cambio $R^{2}=0.030, p<0.001$ ) (véase Tabla 2). De acuerdo con el modelo de trabajo presentado en la Figura 3, el clima de apoyo aumentará el impacto de la comunicación de funciones directivas en la reducción del conflicto de rol. Estos datos vienen a confirmar los argumentos defendidos por diferentes autores (Babin \& Boles, 1996; House \& Rizzo, 1972; Teas, 1983; Shanoch \& Eisenberger, 2006) que han demostrado la relación existente entre la percepción de apoyo y diferentes variables relacionadas con el estado emocional de los trabajadores y el conflicto de rol. 


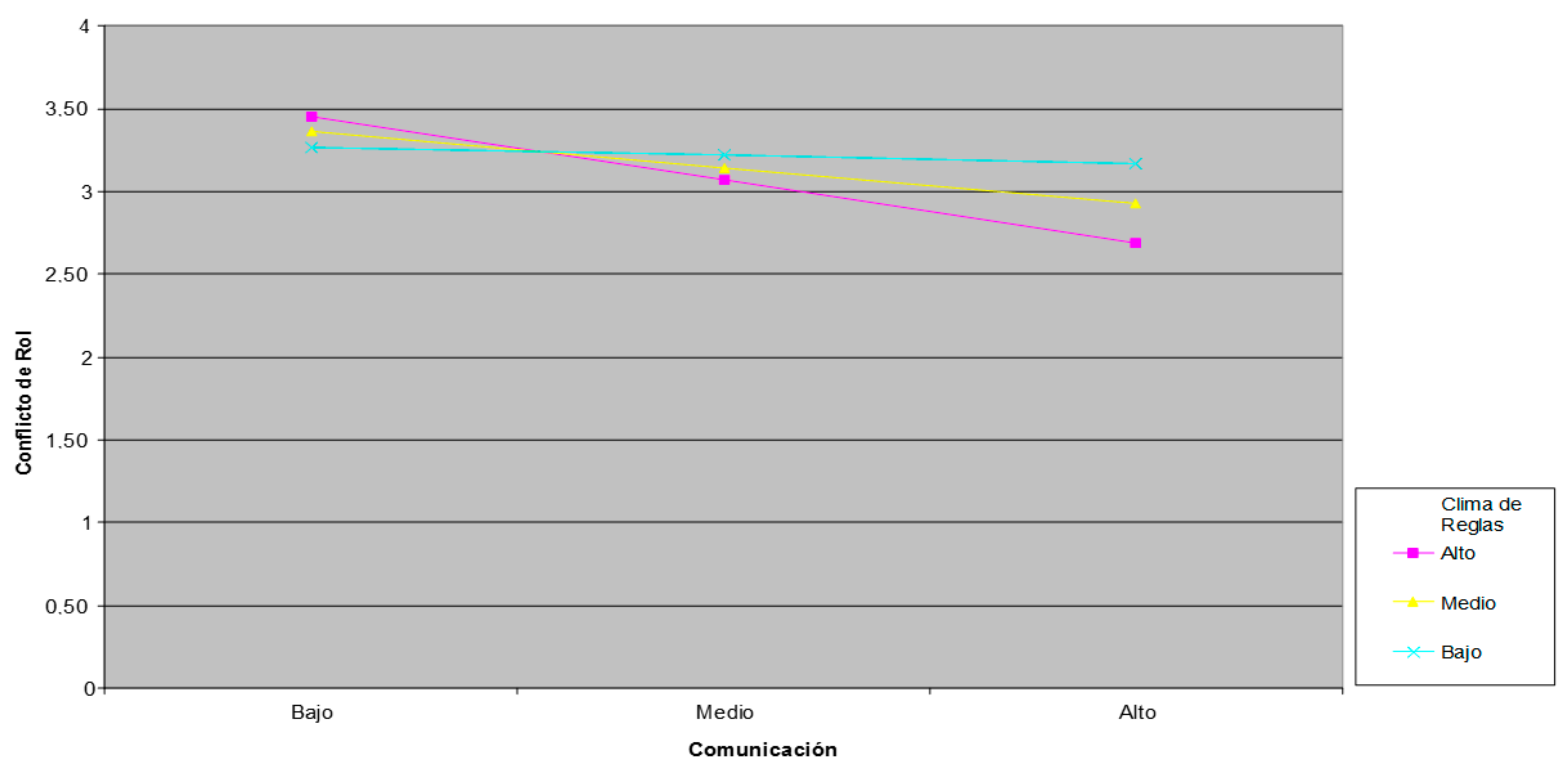

Figura 5. Efectos de modulación del clima de orientación a reglas sobre la relación entre comunicación de funciones directivas y conflicto de rol

Fuente: elaboración propia

Respecto a la segunda hipótesis, que predecía el efecto modulador del clima de innovación sobre la relación de influencia entre la comunicación de funciones directivas y el conflicto de rol, los resultados obtenidos confirman la hipótesis (cambio $R^{2}=0.016 ; p<0.05$ ) (véase Tabla 3). Otros autores como Aarons y Sommerfeld (2012) mostraron que los esfuerzos de los líderes para cambiar las actitudes de los trabajadores se tienen que ver reforzados por la existencia de un clima de innovación adecuado.

La tercera de las hipótesis, que predecía el efecto modulador del clima de orientación a metas sobre la relación e influencia entre la comunicación de funciones directivas y el conflicto de rol, es rechazada por los resultados obtenidos al no mostrarse ningún efecto modulador. Kabiri, Hughes y Schweber (2012) hallaron resultados similares en un estudio sobre el conflicto y la ambigüedad de rol. En su caso, el aumento de la comunicación organizacional y la orientación hacia las metas organizacionales aumentaron los niveles de conflicto de rol. Esto lo explican como efecto del aumento de la claridad de metas organizacionales cuando estás son contrarias con las expectativas sobre las mismas que tienen los trabajadores. Así, una explicación a este resultado es que el aumento de la comunicación del líder, asociado al aumento de los niveles de orientación a metas, puede no reducir los niveles de conflicto de rol en los empleados o incluso aumentarlos en función de las expectativas previas de los trabajadores sobre las metas organizacionales.

Por último, respecto a la hipótesis cuarta, que planteaba la existencia de un efecto modulador del clima de orientación a reglas entre la relación e influencia de la comunicación de funciones directivas y el conflicto de rol, los resultados confirmaron la hipótesis (cambio $R^{2}=0.035 ; p<0.001$ ) (véase Tabla 5).

Para finalizar, los resultados confirman el modelo teórico planteado por Albrecht et al. (2015), al demostrar la influencia de las prácticas de gestión recursos humanos, en este caso, el comportamiento 
de los líderes respecto a la comunicación, y el clima organizacional sobre los estados cognitivos y afectivos de los trabajadores.

\section{Limitaciones del estudio}

Estos resultados deben ser tratados con cautela debido a las limitaciones del presente estudio. La primera de estas restricciones viene dada por la muestra, esta se encuentra muy localizada tanto a nivel geográfico, en la provincia de Almería (España), como del sector productivo, centrado en las cooperativas vinculadas a la hortofruticultura.

En segundo lugar, el uso exclusivo de medidas de autoinforme plantea el problema de la varianza común del método, es decir, la varianza atribuible a método de medida más que a variables consideradas.

En tercer lugar, hay que tener en cuenta que el uso de un diseño transversal también presenta una limitación, ya que no permite establecer relaciones de tipo causal.

Futuras líneas de investigación [t3]

Para superar estas tres limitaciones en posteriores estudios sería necesario que incluir otras muestras más heterogéneas en cuanto a su ubicación y sector, utilizando metodologías de análisis longitudinales con medidas repetidas e incorporar otras metodologías de recogida de información que permitan multiplicar los datos a analizar y los contrastes que se puedan realizar.

\section{Implicaciones prácticas}

La principal aportación práctica radica en el papel relevante que ocupa el clima organizacional entre los comportamientos de los líderes de la organización y la prevención, así como en la reducción del conflicto de rol de los trabajadores.

También se destaca el cuidado que debe haber de las conductas y comportamientos de los líderes organizacionales, ya que del impacto que las mismas producen en los trabajadores dependerá en buena medida la mejora de la eficiencia organizacional. (Haakonsson, Burton, Obel, \& Lauridsen, 2008; Walumbwa, Hartnell, \& Oke, 2010; Wang \& Rode, 2010).

\section{Referencias}

Aarons, G. A., \& Sommerfeld, D. H. (2012). Leadership, Innovation Climate, and Attitudes Toward Evidence-Based Practice During a Statewide Implementation. Journal of the American academy of child and adolescent psychiatry, 51, 423-431.

Albrecht, S. L., Bakker, A. B., Gruman, J. A., Macey, W. H., \& Saks, A. M. (2015). Employee engagement, human resource management practices and competitive advantage: An integrated approach. Journal of Organizational Effectiveness: People and Performance, 2, 7-35.

Babin, B. J., \& Boles, J. S. (1996). The effects of perceived co-worker involvement and supervisor support on service provider role stress, performance and job satisfaction. Journal of Retailing, 72, 57-75.

Calvete, E. (2008). Una introducción al análisis de moderación y mediación: Aplicaciones en el ámbito del estrés. Anales de Psicología, 14, 159-173.

Cohen, J., Cohen, P., West, S. G., \& Aiken, L. S. (2003). Applied multiple regression/correlation analysis for the behavioral sciences. Mahwah, NJ: Erlbaum.

De Witte, K., \& De Cock, G. (1986). Organizational climate: Its relation with managerial activities or communication structures. In G. Debus, \& H. W. Schroiff (Eds.), The psychology of work and organization: Current trends and issues (pp.70-96). Amsterdam: North Holland.

Frazier, R. A., Tix, A. P., \& Barron, K. E. (2004). Testing moderator and mediator effects in counseling psychology research. Journal of Counseling Psychology, 51, 115-134.

Haakonsson, D. D., Burton, R. M., Obel, B., \& Lauridsen, J. (2008). How failure to align organizational climate and leadership style affects performance. Management Decision, 46, 406-432.

House, R. J., \& Rizzo, J. R. (1972). Role conflict and ambiguity as critical variables in a model of organizational behavior. Organizational behavior and Human Performance, 1, 467-505.

Johlke, M. C. \& Duhan, D. F. (2001). Supervisor communication practices and boundary spanner role ambiguity. Journal of Managerial Issues, 13, 87-101.

Kabiri, S, Hughes, W., \& Schweber, L. (2012). Role conflict and role ambiguity in construction projects 
In S. D. Smith (Ed.), Procs 28th Anmual ARCOM Conference, 3-5 September 2012, Edinburgh, UK, Association of Researchers in Construction Management, 727-736.

Kopelman, R. E., Brief, A. P., \& Guzzo, R. A. (1990). The role of climate and culture in productivity. En B. Schneider (Ed.), Organizational climate and culture (pp. 282-318). San Francisco: Jossey-Bass.

Likert, R. (1961) New patterns of management. New York: McGraw Hill.

MacKenzie, C. A., Garavan, T. N., \& Carbery, R. (2012). Through the looking glass: challenges for human resource development (HRD) post the global financial crisis-business as usual? Human Resource Development International, 15, 353-364.

McGregor, D. (1960). The human side of enterprise. New York: McGraw Hill.

McMurray, A. J., Islam, M., Sarros, J. C., \& Pirola-Merlo, A. (2012). The impact of leadership on workgroup climate and performance in a non-profit organization. Leadership $\mathcal{E}$ Organization Development Journal, 33, 522-549.

Peiró, J. M., Meliá, J. L., Torres M. A., \& Zurriaga R. (1986). La medida de la experiencia de la ambigüedad en el desempeño de roles: el cuestionario general de ambigüedad de rol en ambientes organizacionales. Evaluación psicológica, 3, 27-53.

Peiró, J. M., \& González-Romá, V. (dirs.) (1994). Estudio sobre los correlatos psicológicos del absentismo en el personal del Servicio Valenciano de Salud. Valencia, España: Universitat de Valencia. Unidad de Investigación de Psicología de las Organizaciones y del Trabajo (UIPOT).

Pérez, F. J. P., Guzmán, T. L. G., \& Santa Cruz, F. G. (2014). Management skills as competitive advantage. The case of public sector in Córdoba (Spain). Intangible Capital, 10, 528-561.

Quinn, R. E., \& Rohrbaugh, J. (1983). A spatial model of effectiveness criteria: Towards a competing values approach to organizational analysis. Management Science, 29, 363-377.

Rizzo, J. R., House, R. J., \& Lirtzman, I. (1970). Role conflict and ambiguity in complex organizations. Administrative Science Quarterly, 15, 150-163.

Salanova, M., Llorens, S., Cifre, E., \& Martínez, I. M. (2012). We need a hero! Toward a validation of the healthy and resilient organization (HERO) model. Group E Organization Management, 37, 785-822.

Schneider, B., \& Reichers, A. E. (1983). On the etiology of climates. Personnel Psychology, 36, 19-39

Teas, R. K. (1983). Supervisory behavior, role stress, and the job satisfaction of industrial sales people. Journal of Marketing Research, 20, 84-91.

Tomasina, F. (2012). Los problemas en el mundo del trabajo y su impacto en salud. Crisis financiera actual. Revista de Salud Pública, 14, 56-67.

Van Muijen, J. J., Koopman P., De Witte, K., De Cock, G., Susanj Z., Lemoine C., ... Turnipseed, D. (1999). Organizational culture: The focus questionnaire. European Journal of Work and Organizational Psychology, 8, 551-568.

Walumbwa, F. O., Hartnell, C. A., \& Oke, A. (2010). Servant leadership, procedural justice climate, service climate, employee attitudes, and organizational citizenship behavior: A cross-level investigation. Journal of Applied Psychology, 95, 517-529.

Wang, P., \& Rode, J. C. (2010). Transformational leadership and follower creativity: The moderating effects of identification with leader and organizational climate. Human Relations, 63, 1105-1128

Yukl, G. (2006). Leadership in organizations (6 Ed.). Upper Saddle River, NJ: Prentice Hall.

Yukl, G., \& Van Fleet, D. (1992) Theory and research on leadership in organizations. In M. D. Dunnette, \& L. M. Hough (Eds.), Handbook of Industrial and Organizational Psychology, Vol. 3 (pp. 147-197). Palo Alto, CA: Consulting Psychologists Press. 
